\title{
A Comparative Study of Bone Resorption between Implant Placement Sites and Non-Implant Placement Sites after Autogenous Block Bone Grafts in the Anterior Maxilla
}

\author{
Jisi Zheng1, Shanyong Zhang1, Huaihai Fan², Eryi Lu³, Chi Yang1, Jingyang Zhao4 \\ ${ }^{1}$ Department of Oral and Maxillofacial Surgery, Ninth People's Hospital, Collage of Stomatology, Shanghai Jiao \\ Tong University School of Medicine, Shanghai, China \\ ${ }^{2}$ The Intensive Care Unit, The Central Hospital of Taian, Qingdao, China \\ ${ }^{3}$ Department of Prosthodontics, Ninth People's Hospital, Collage of Stomatology, Shanghai Jiao Tong University \\ School of Medicine, Shanghai, China \\ ${ }^{4}$ Department of Oral Implantology, Hospital Affiliated Qingdao University School of Medicine, Qingdao, China \\ Email: zhangshanyong@126.com
}

Received 5 March 2014; revised 8 April 2014; accepted 16 April 2014

Copyright (C) 2014 by authors and Scientific Research Publishing Inc.

This work is licensed under the Creative Commons Attribution International License (CC BY). http://creativecommons.org/licenses/by/4.0/

\section{Open Access}

\section{Abstract}

The aim of this study was to compare the bone resorption differences between implant placement sites (IPS) and non-implant placement sites (NIPS) after autogenous block bone grafts in the anterior maxilla. Fourteen patients (58 edentulous sites) with alveolar atrophy in the anterior maxilla were treated with autogenous block bone grafts. CBCT examinations were performed at 1 month before surgery (T0), immediately after surgery (T1), 3 to 4 months after surgery (T2), 6 to 7 months after surgery before implant placement (T3), 12 to 13 months after surgery (T4), and the longest follow-up point (T5). Alveolar crestal and basal bone width (ACBW, ABBW), and alveolar bone height (ABH) were measured and divided into IPS (30 sites) and NIPS (28 sites). All results were compared by the Wilcoxon Signed Rank test. The bone resorption changes for both groups were the same. For these three parameters, ACBW didn't change significantly from T2 to T3 and T4 to T5, ABBW didn't change at every period from T2 to T5, and ABH didn't change from T4 to T5. The bone resorption volume of ACBW and $A B H$ in NIPS were more than in IPS after implant placement surgery, while the volume of ABBW was similar in both groups. At T5, the bone resorption percentages of ACBW, ABBW, and ABH were $25.57 \%, 16.85 \%$ and $43.84 \%$ in IPS, and $33.55 \%$, $\mathbf{1 5 . 9 2 \%}$ and $46.44 \%$ in NIPS. A more rapid loss of alveolar crest in NIPS resulted from implant placement surgery, and this reminded us of the importance of immediate implant placement. 


\section{Keywords}

\section{Implant, Anterior Maxilla, Bone Resorption, Implant Placement Site, Non-Implant Placement Site}

\section{Introduction}

The bone deficiency in the anterior maxilla resulting from teeth loss, trauma, and periodontal disease, remains a major challenge for both the surgeons and prosthodontists [1]. Since the principles of osteogenesis, osteoconduction, and osteoinduction can be used to optimize therapeutic approaches [2], several approaches, including autogenous bone graft, allograft and xenograft (as block or particulate form), which have been explored for different surgical procedures [3] [4]. Among the techniques used to reconstruct the reduced residual bone, autogenous block bone grafts has become a widely accepted method to regain the bone volume for implant placement [5] [6]. It is no doubt that the main purpose of bone grafts is to obtain natural tissue contours in preparation for the proposed implant prosthesis [7]. However, bone loss occurs after surgery is difficult to predict, so it becomes a central issue that has been studied in recent years [7]-[9].

It is necessary to know that the bone quality and quantity of the anterior maxilla after teeth loss will become thinner and lower according to Lekholm and Zarb (1985) classification [10]. The emphases in recent studies have focused on the differences among: 1) the bone width and height resorption [5] [11]; 2) the block bone and particulate bone [12] [13]; 3) the titanium mesh and collagen membrane, and centered on the influences of different: a) bone materials [12] [14]; b) growing factors for bone formation or regeneration [15] [16]. However, there were also several studies to discuss the bone preservation due to implant placement. Cosyn et al. [17] showed that a correct implant and contact point positioning could optimize soft and hard tissue levels around a single implant. Tomohiro et al. [18] also compared the vertical bone resorption changes between implant-implant and implant-pontic restorative environment. The implant under pontic restorative position was used to prevent the bone resorption after bone grafts. It is worth mentioning that Dasmah et al. [19] put forward that the implant protected the grafts from continuous vertical resorption, and the resorption in non-implant areas was prolonged to a greater extent. Therefore, implant, just like natural teeth, could prevent bone resorption and obtain natural soft and hard tissue contours [20]. Therefore, the sooner implant may be placed in the anterior maxilla, the better the alveolar bone may be preserved [21]. However, there were still no studies to compare the bone resorption differences between implant areas and non-implant areas.

In conclusion, the aim of the present study was to conduct a comparative study of implant placement sites (IPS) vs. non-implant placement sites (NIPS), using cone beam computed tomography (CBCT) scans. We compared the alterations of the alveolar crestal bone width (ACBW), alveolar basal bone width (ABBW) and alveolar bone height $(\mathrm{ABH})$ after autogenous block bone grafts in the anterior maxilla over a period of 20.3 months [5].

\section{Materials and Methods}

\subsection{Patients}

A total of 14 consecutive patients (58 edentulous sites) who visited the department of oral surgery at the Ninth People's Hospital, Shanghai Jiao Tong University School of Medicine, Shanghai, China, from May 2010 to August 2011, were selected. All patients were in accordance with the following requirements: 1) multiple and continuous teeth loss in the anterior maxilla; 2$)$ anterior alveolar bone height defects $(=<5 \mathrm{~mm})$ and residual bone width $(=<6 \mathrm{~mm})$ [9], according to CBCT (NewTom VG QR, Verona, Italy) scans at 1 month before surgery (T0); 3) teeth loss for more than 6 months [22]. There were seven male and seven female patients with a mean age of 32.7 years (range, 27 - 38 years). The longest available mean follow-up was 20.3 months (range, 18 - 22 months). There were 2 cases due to trauma and 12 cases due to periodontal disease. The baseline characteristics of all the patients were as shown in Table 1. This study was conducted in accordance with the Ethics Committee of Shanghai Jiao Tong University School of Medicine.

\subsection{Preoperative Surgical Care}

Antibiotics made up of both cefradine $(250 \mathrm{mg} \times 2$ ) and ornidazole $(250 \mathrm{mg} \times 2)$ were given during $24 \mathrm{~h}$ before 
Table 1. The baseline characteristics of all the patients.

\begin{tabular}{ccccccc}
\hline \multirow{2}{*}{ Number } & \multicolumn{7}{c}{ The baseline characteristics of the patients } \\
\cline { 2 - 7 } & Age (years) & Sex & Edentulous sites & IPS & NIPS & Follow-up (months) \\
\hline $\mathbf{1}$ & 27 & M & $12-22$ & 12,22 & 11,21 & 18.3 \\
$\mathbf{2}$ & 29 & F & $11-22$ & 11,22 & 21 & 21.9 \\
$\mathbf{3}$ & 38 & M & $13-23$ & $13,12,23$ & $11,21,22$ & 19.7 \\
$\mathbf{4}$ & 34 & M & $11-22$ & 11,22 & 21 & 20.1 \\
$\mathbf{5}$ & 28 & F & $12-22$ & 12,22 & 11,21 & 21 \\
$\mathbf{6}$ & 32 & M & $12-23$ & $12,22,23$ & 11,21 & 19.8 \\
$\mathbf{7}$ & 33 & F & $13-23$ & 13,23 & $12-22$ & 19 \\
$\mathbf{8}$ & 35 & F & $12-22$ & 12,22 & 11,21 & 21.1 \\
$\mathbf{9}$ & 29 & M & $13-11$ & 13,11 & 12 & 22 \\
$\mathbf{1 0}$ & 33 & F & $11-23$ & 11,23 & 21,22 & 18 \\
$\mathbf{1 1}$ & 37 & M & $12-22$ & 12,22 & 11,21 & 21.2 \\
$\mathbf{1 2}$ & 36 & F & $13-21$ & 13,21 & 11,12 & 21.3 \\
$\mathbf{1 3}$ & 31 & F & $11-23$ & 11,23 & 21,22 & 20.5 \\
$\mathbf{1 4}$ & 36 & M & $12-22$ & 12,22 & 11,21 & $\mathbf{2 0 . 3}$ \\
\hline
\end{tabular}

bone grafts. All patients received ornidazole $(250 \mathrm{mg} \times 2)$ before implant placement as a single dose.

\subsection{Surgical Procedure}

Under local anesthesia, cortico-cancellous block bone was harvested from either the chin or mandibular ramus by using a triphine drill (Osstem, Seoul, South Korea). A high speed, water-cooled, round bur was used to shape the harvested block bone before delivery to the recipient site. The anterior maxilla was freed from the periosteum and prepared with the previously mentioned round bur until small spots of bleeding were noted. Then, block bone was adjusted to fit the anatomy of the recipient site and secure to the underlying recipient bone with a titanium screw (OsteoMed, Addison, TX) without any possibility of micromovement. An additional mixture of Bio-Oss particle (Geistlich Biomaterials, Wolhusen, Switzerland) and blood obtained from the recipient site was placed at the periphery of the block bone. The recipient site was further protected with Bio-Gide collagen membrane (Geistlich Biomaterials, Wolhusen, Switzerland). Then, the buccal flap of soft tissue was elongated through small incisions of the periosteum to gain full and tension-free coverage of the grafted areas. The incisions were closed with resorbable sutures (Vicryl, Sollentuna, Sweden) as shown in Figure 1.

During 6 to 7 months after surgery, implants (30 implants in total) (NobelReplace, Sollentuna, Sweden) were installed with the help of an implant guide under the mucoperiosteal flap reflection, which was used to expose the alveolar crest of the recipient site, not as well as previously stated mucoperiosteal flap reflection as shown in Figure 2.

\subsection{Postoperative Surgical Care}

All Patients also received a prophylactic antibiotic cure during 10 days after block bone grafts, with both cefradine $(250 \mathrm{mg} \times 2)$ and ornidazole $(250 \mathrm{mg} \times 2)$. Patients received ornidazole $(250 \mathrm{mg} \times 2)$ during 7 days after implant placement. Lamiophlomis $(300 \mathrm{mg} \times 3)$ and acetaminophen $(250 \mathrm{mg} \times 2)$ were given as analgesic hemostasis for 7 days after block bone grafts and implant placement.

\subsection{Postoperative Evaluation}

Wound healing, general condition, implant survival situation, and so on, were evaluated after block bone grafts and implant placement [11]. CBCT scans for all patients were obtained again immediately after surgery (T1), 3 to 4 months after surgery (T2), 6 to 7 months after surgery before implant placement (T3), 12 to 13 months after 


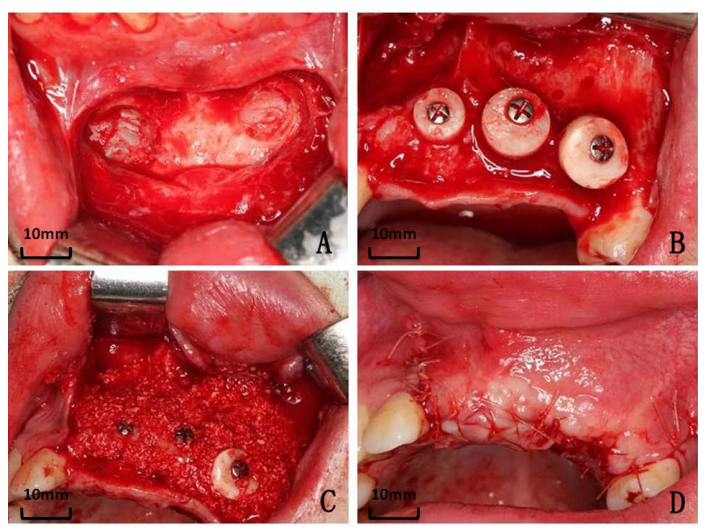

Figure 1. Autogenous block bone grafts. A, The donor site. B, Autogenous block bone grafts. C, Bio-Oss grafts. D, Suture of the wound.
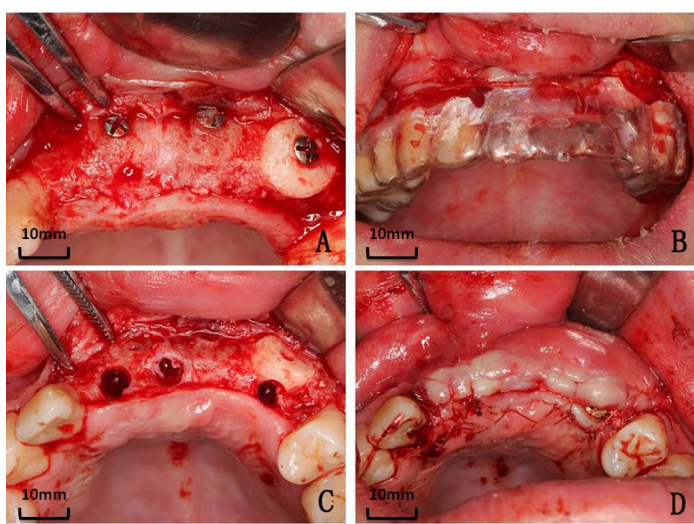

Figure 2. Implant placement surgery. A, The mucoperiosteal flap reflection. B, Implant guide. C, Implant placement. D, Suture of the wound.

surgery (T4), and the longest postoperative follow-up point (T5) [5]. This radiographic measurement method reported by Block et al. in 2012 was selected [5]. The data from CBCT scans in the DICOM (Digital Imaging and Communications in Medicine) format were input into an interactive Simplant software program (Version 11.04, Materialise Medical, Leuven, Belgium) to reconstruct all patients' 3-Dimensional surface model. The maxillary occlusal plane was adjusted parallelly to the horizontal plane. The virtual teeth function in the Simplant software was used to repair the edentulous dentition. Then the virtual teeth axis was determined to be the measurement slice so that each measurement was as close to the same slice possible. Referring to other relative articles [5] [23], three following parameters were selected to measure: 1) alveolar crestal bone width (ACBW): the distance from labial to palatal at the alveolar crest; 2) alveolar basal bone width (ABBW): the distance from labial to palatal or lingual at the alveolar base; and 3) alveolar bone height (ABH): the distance from alveolar crest to basal bone. All results were divided into two groups: implant placement sites (IPS) and non-implant placement sites (NIPS). These three parameters were measured twice at every slice by different surgeons as shown in Figure 3.

\subsection{Statistical Analysis}

The Wilcoxon Signed Rank test in the SAS software package (version 9.0, SAS Institute, Cary, NC), was used for statistical analysis of the previously stated three parameters. The results included the following contents: 1) the bone resorption changes for both groups over time; 2) the bone resorption differences for both groups after autogenous block bone grafts and implant placement; and 3) the bone resorption percentages for both groups at every follow-up point. There was a significant difference of $\mathrm{P}<0.05$. 

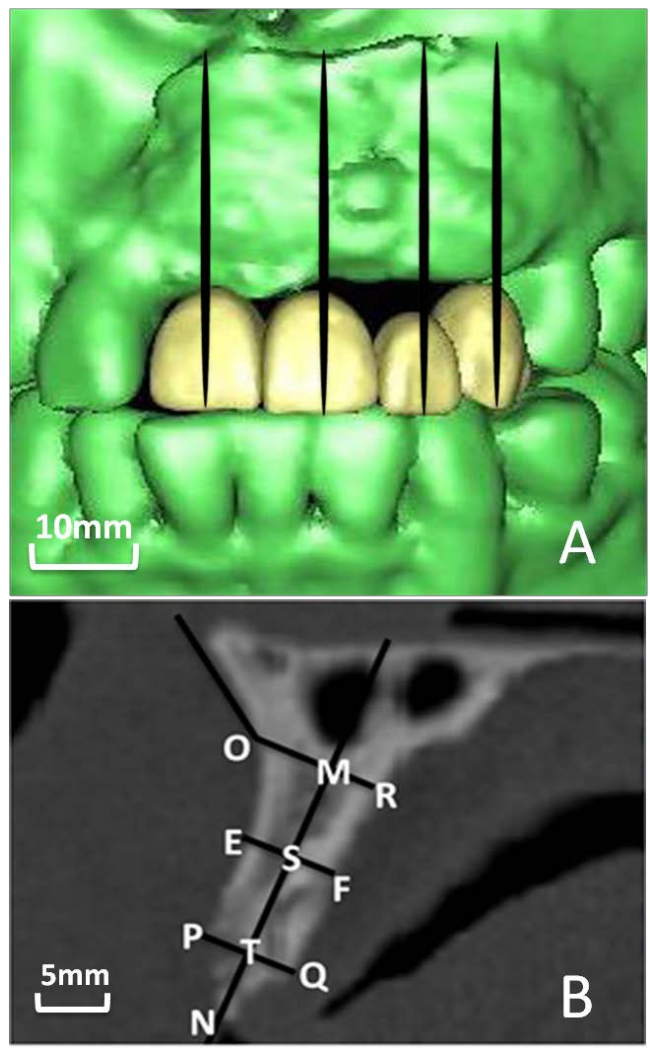

Figure 3. The measurement method. A, The virtual teeth function in Simplant software. B, The standardization of the "baseline" measurements; point $\mathrm{O}$, measurement from the tip of the anterior nasal spine to the concavity on the buccal cortex; point $\mathrm{S}$, the middle point of line $\mathrm{MT}$; line $\mathrm{MN}$, paralleling lingual cortex; line OR, vertical to $\mathrm{MN}$ and alveolar basal bone width; line PQ, vertical to $\mathrm{MN}$ and alveolar crestal bone width; line EF, vertical to $\mathrm{MN}$; line $\mathrm{MN}$, the alveolar bone height.

\section{Results}

\subsection{General Situation}

All block bone grafts and implant placement surgeries were completed successfully. Dizziness, headache, and other uncomfortable conditions did not occur and the incisions healed excellently. Successful restorations were achieved in all patients with fixed implant-supported prostheses, and all implants remained clinically osseointegrated at the longest postoperative follow-up point.

\subsection{The Bone Resorption Changes of These Three Parameters}

The bone resorption changes for both groups were the same. For these three parameters respectively, listed in Table 2 (IPS) and Table 3 (NIPS), there were significant differences for ACBW from T1 to T2 and T3 to T4, for ABBW from $\mathrm{T} 1$ to $\mathrm{T} 2$, and for $\mathrm{ABH}$ from $\mathrm{T} 1$ to $\mathrm{T} 2$, $\mathrm{T} 2$ to $\mathrm{T} 3$ and $\mathrm{T} 3$ to $\mathrm{T} 4$. In addition, there were no significant differences for ACBW from T2 to T3 and T4 to T5, for ABBW from T2 to T3, T3 to T4, and T4 to T5, and for $\mathrm{ABH}$ from $\mathrm{T} 4$ to $\mathrm{T} 5$.

\subsection{The Differences of Bone Resorption Volume for Both Groups}

The bone resorption differences for both groups, drawn in Figure 4, were as follows: 1) there were no signifi- 
Table 2. Comparison of bone augmentation and bone resorption in IPS (mm).

\begin{tabular}{rcccccc}
\hline & \multicolumn{2}{c}{ ACBW } & \multicolumn{2}{c}{ ABBW } & \multicolumn{2}{c}{ ABH } \\
\cline { 2 - 7 } & Mean \pm STD (mm) & P Value & Mean \pm STD (mm) & P Value & Mean \pm STD (mm) & P Value \\
\hline T1 - T0 & $5.3423 \pm 0.8042$ & $<0.0001^{*}$ & $6.1202 \pm 0.9622$ & $<0.0001^{*}$ & $5.9455 \pm 0.9887$ & $<0.0001^{*}$ \\
T1 - T2 & $0.8398 \pm 0.7772$ & $<0.0001^{*}$ & $0.7156 \pm 0.8464$ & $<0.0001^{*}$ & $1.4273 \pm 0.6827$ & $<0.0001^{*}$ \\
T2 - T3 & $0.0977 \pm 0.6105$ & 0.2202 & $0.1498 \pm 0.5305$ & 0.0712 & $0.2887 \pm 0.5354$ & $<0.0001^{*}$ \\
T3 - T4 & $0.3735 \pm 0.5781$ & $<0.0001^{*}$ & $0.1347 \pm 0.8732$ & 0.3177 & $0.5378 \pm 0.5475$ & $<0.0001^{*}$ \\
T4 - T5 & $0.0548 \pm 0.4305$ & 0.3278 & $0.0309 \pm 0.1181$ & 0.0932 & $0.0353 \pm 0.1841$ & 0.1424 \\
\hline
\end{tabular}

Note: The bone augmentation (from T0 to T1) and resorption (from T1 to T2, T2 to T3, T3 to T4, and T4 to T5) in IPS were compared by the Wilcoxon Signed Rank test in the SAS software package, and there was a significant difference of $\mathrm{P}<0.05,{ }^{*} \mathrm{P}<0.05$.

Table 3. Comparison of bone augmentation or bone resorption in NIPS (mm).

\begin{tabular}{ccccccc}
\hline & \multicolumn{2}{c}{ ACBW } & \multicolumn{2}{c}{ ABBW } & \multicolumn{2}{c}{ ABH } \\
\cline { 2 - 7 } & Mean \pm STD (mm) & P Value & Mean \pm STD (mm) & P Value & Mean \pm STD (mm) & P Value \\
\hline T1 - T0 & $5.3150 \pm 0.7591$ & $<0.0001^{*}$ & $6.1693 \pm 0.9146$ & $<0.0001^{*}$ & $6.0582 \pm 0.8510$ & $<0.0001^{*}$ \\
T1 - T2 & $0.7554 \pm 0.7948$ & $<0.0001^{*}$ & $0.7216 \pm 0.8028$ & $<0.0001^{*}$ & $1.2918 \pm 0.5629$ & $<0.0001^{*}$ \\
T2 - T3 & $0.1086 \pm 0.5431$ & 0.1404 & $0.1193 \pm 0.4990$ & 0.0791 & $0.3061 \pm 0.5079$ & $<0.0001^{*}$ \\
T3 - T4 & $0.8464 \pm 0.5566$ & $<0.0001^{*}$ & $0.1116 \pm 0.7760$ & 0.2865 & $1.1704 \pm 0.7725$ & $<0.0001^{*}$ \\
T4 - T5 & $0.0730 \pm 0.3596$ & 0.1342 & $0.0296 \pm 0.1393$ & 0.1170 & $0.0454 \pm 0.2310$ & 0.1474 \\
\hline
\end{tabular}

Note: The bone augmentation (from T0 to T1) and resorption (from T1 to T2, T2 to T3, T3 to T4, and T4 to T5) in NIPS were compared by the Wilcoxon Signed Rank test in the SAS software package, and there was a significant difference of $\mathrm{P}<0.05,{ }^{*} \mathrm{P}<0.05$.

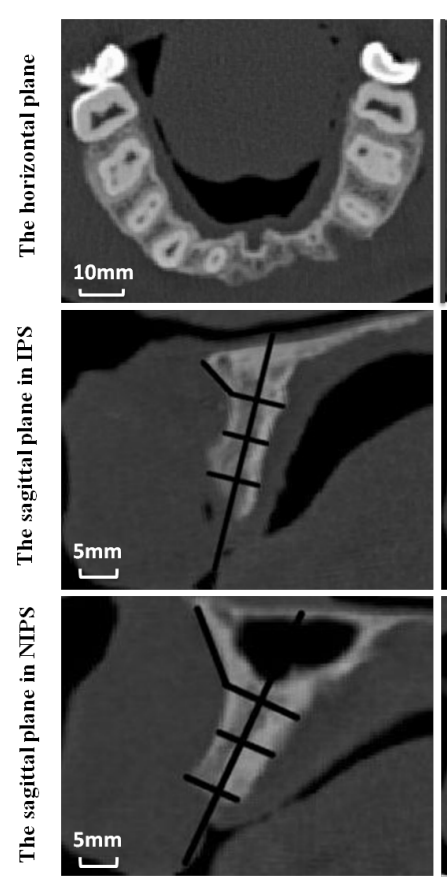

1 month before surgery
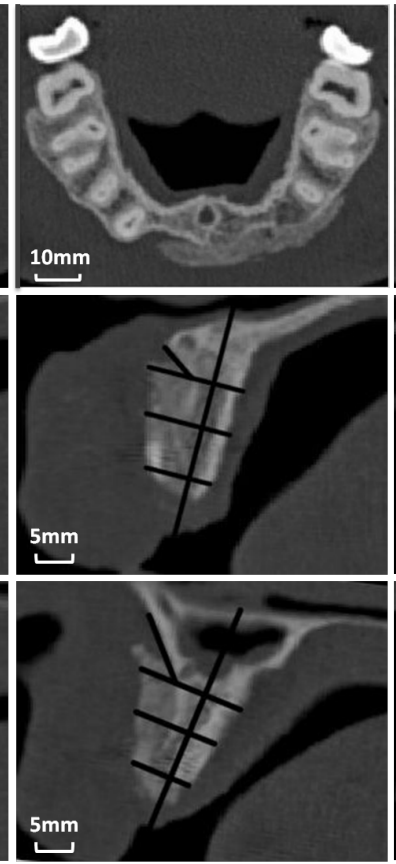

Immediately after surgery

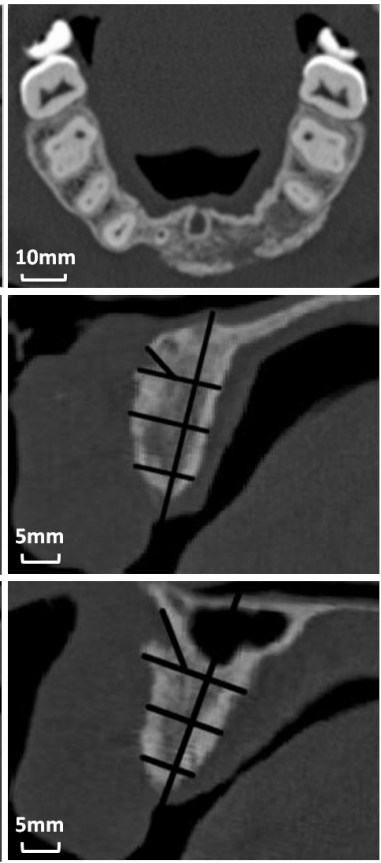

6 to 7 months after surgery

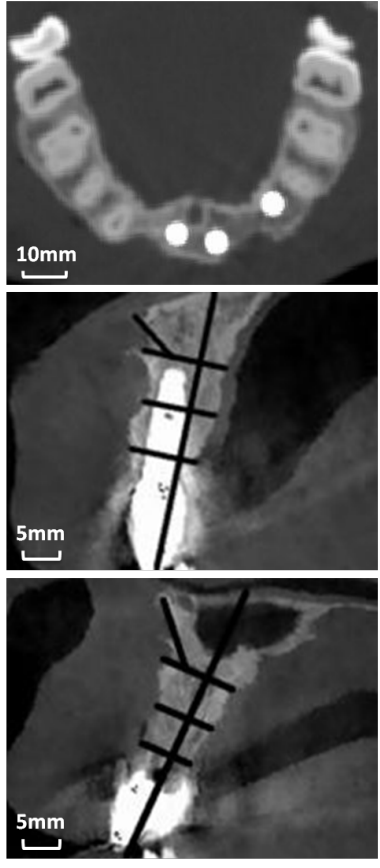

The longest follow-up point

Figure 4. The bone resorption differences between IPS and NIPS at the different follow-up points.

cant differences for ACBW and $\mathrm{ABH}$ at T0, T1, T2, and T3, but there were significant differences at T4 and T5;

2) there were no significant differences for ABBW at every postoperative follow-up point as shown in Figure 5. 

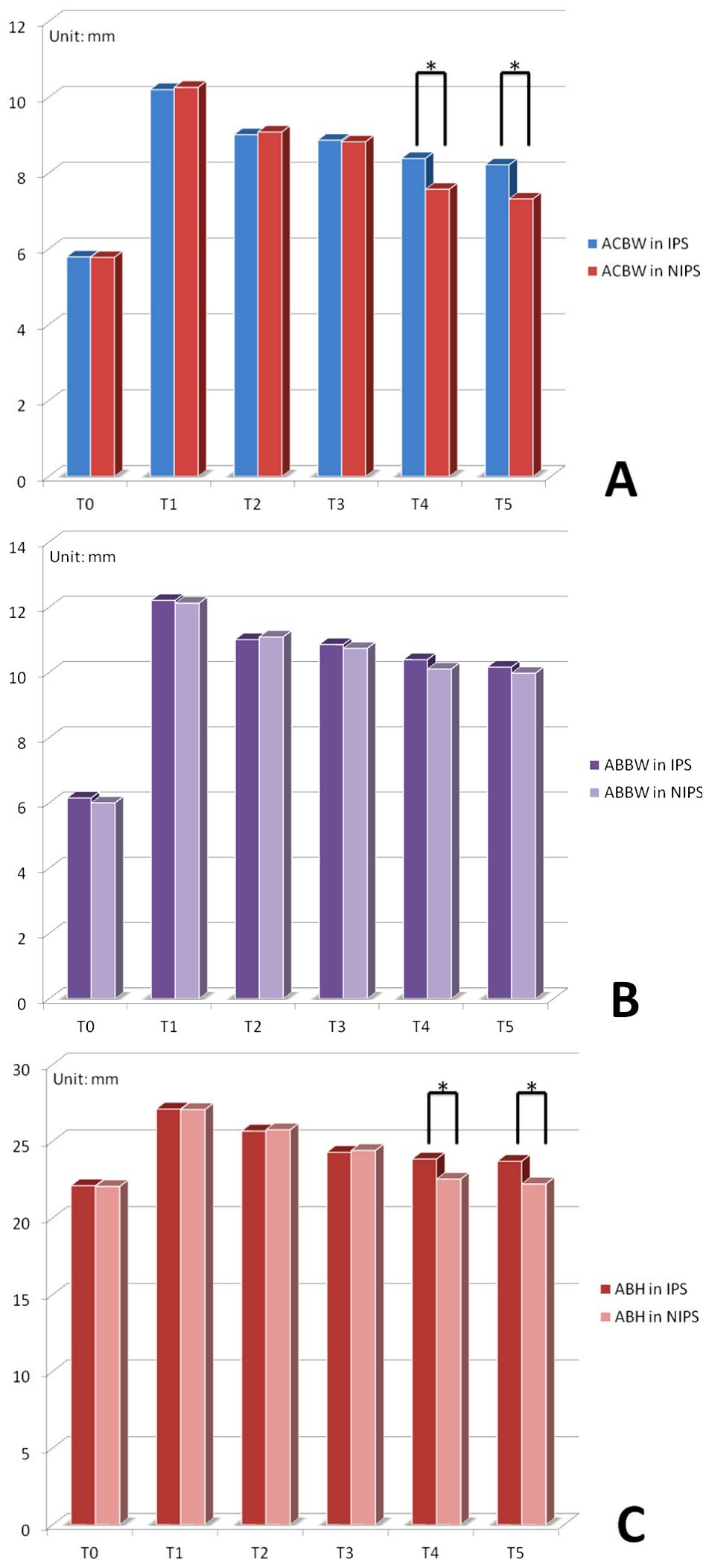

Figure 5. Comparison of the bone augmentation and resorption. A, ACBW in IPS and ACBW in NIPS. B, ABBW in IPS and ABBW in NIPS. $C, A B H$ in IPS and ABH in NIPS. ${ }^{*} \mathrm{P}<0.05$. 


\subsection{The Bone Resorption Percentages of These Three Parameters}

The bone resorption percentages of these three parameters at every follow-up point were listed in Table 4. It was most noteworthy that the bone resorption percentages for ACBW, ABBW, and ABH, at the longest postoperative follow-up point, were $25.57 \%, 16.85 \%$ and $43.84 \%$ in IPS, and $33.55 \%, 15.92 \%$ and $46.44 \%$ in NIPS.

\section{Discussion}

The edentulous alveolar process typically underwent extensive resorption in the absence of functional stimulation [3]. Once the anterior maxilla severely atrophied, sometimes leading to facial recession, it was usually necessary for bone grafts to acquire natural soft and hard tissue contours [24]. However, the bone resorption still existed in the bone grafts [25]-[27]. To our knowledge, a considerable amount of radiological studies has used CBCT scans to provide sectional or 3-Dimensional information for the linear or volumetric measurements [28]. The method in which the actual measurements were conducted was important. Therefore, we selected a comprehensive measurement method, which has been reported by Block et al. in 2012 [5]. The advantages of the method included determination of the same measurement slice in each measurement and different evaluation indexes from alveolar crestal and basal bone width and alveolar bone height. Therefore, the method made up the weakness that a possible difference was therefore not possible to detect.

In this study, it was between implant placement sites (IPS) and non-implant placement sites (NIPS) that the importance of alveolar bone preservation due to implant placement was given more attention by evaluating and comparing the bone resorption differences. Even though the bone resorption changes for both groups were the same, the bone resorption volume for both groups became significantly different after implant placement surgery. Furthermore, ABBW did not change significantly since 6 months after surgery, earlier than ACBW and ABH. A previous volumetric study conducted by Nyström et al. [13] has shown that a significant amount of resorption in bone grafts took place during the first 6 months after surgery. Lin et al. [29] has also reported that most of the resorption of onlay bone grafts took place during the first 6 postoperative months. The results of the previously stated studies were the same as the bone resorption changes of ABBW in the present study. However, three questions arise, "Why did the bone resorption volume for both groups became significant different after implant placement surgery?", "Why was there significant bone resorption again for ACBW from 9 to 10 months after surgery, and why did it not happen for ABBW?", and "Why did the bone resorption for ABH not change significantly until the last follow-up point?"

On the one hand, the blood supply of the alveolar crest was blocked due to the mucoperiosteal flap reflection, which resulted in alveolar crestal exposure during implant placement surgery. On the other hand, as mentioned above, implant placement surgery may prevent the bone resorption, just as trees can prevent soil erosion. These two factors together led to the further bone resorption of alveolar crest in NIPS. Von et al. [30] demonstrated that vascular supply played an important role on bone formation and regeneration. Barone et al. [6] demonstrated that a mucoperiosteal flap yielded significantly more negative results with an increased width resorption.

For the alveolar bone height, there was more significant bone resorption for bone height than bone width [13]. It was no doubt that the reconstruction of bone height was more difficult than bone width [27]. Usually, most of the width reduction took place during 1 to 6 months after surgery, while the height reduction occurred mainly 1 to 12 months after surgery. In this study, the bone height did not change significantly until 12 to 13 months after surgery. Nyström et al. [13] (as well as the present study) has shown that there was a more rapid and initial loss of bone height than bone width after autogenous block bone grafts, and the height reduction occurred significantly at 1 year examination. Therefore, the height reduction was more than the width.

Table 4. The bone resorption percentages.

\begin{tabular}{rcccccc}
\hline & \multicolumn{2}{c}{ ACBW } & \multicolumn{2}{c}{ ABBW } & \multicolumn{2}{c}{ ABH } \\
\cline { 2 - 7 } & IPS & NIPS & IPS & NIPS & IPS & NIPS \\
\hline T2 & $15.72 \%$ & $14.21 \%$ & $11.69 \%$ & $11.70 \%$ & $24.01 \%$ & $21.32 \%$ \\
T3 & $17.55 \%$ & $16.26 \%$ & $14.14 \%$ & $13.63 \%$ & $28.86 \%$ & $26.38 \%$ \\
T4 & $24.54 \%$ & $32.18 \%$ & $16.34 \%$ & $15.44 \%$ & $37.91 \%$ & $45.70 \%$ \\
T5 & $25.57 \%$ & $33.55 \%$ & $16.85 \%$ & $15.92 \%$ & $43.84 \%$ & $46.44 \%$ \\
\hline
\end{tabular}


This study also listed the bone resorption percentages according to the different sites and follow-up points. The highest bone resorption percentage (46.44\%) was the ABH of NIPS at the longest postoperative follow-up point, while the lowest bone resorption percentage (11.69\%) was the ABBW of IPS at 3 to 4 months after surgery. The results were similar with most recent studies (7.2\% to 52\%) [7] [31]. However, the bone percentages were more beneficial to the comparison with other studies.

In conclusion, this study illustrated the bone resorption differences between IPS and NIPS. This also reminded us of the importance of bone grafts in combination with immediate implant placement.

\section{Acknowledgements}

This study was supported by Research Fund of Science and Technology Committee of Shanghai Municipality (134119a5400) and the Seventh College Students Innovation Training Program of Shanghai Jiao Tong University School of Medicine (2013061).

\section{References}

[1] Isaksson, S. and Alberius, P. (1992) Maxillary Alveolar Ridge Augmentation with Onlay Bone-Grafts and Immediate Endosseous Implants. Journal of Cranio-Maxillofacial Surgery, 20, 2-7. http://dx.doi.org/10.1016/S1010-5182(05)80187-8

[2] Hollinger, J.O., Brekke, J. and Gruskin, E. (1996) Role of Bone Substitutes. Clinical Orthopaedics and Related Research, 324, 55-65. http://dx.doi.org/10.1097/00003086-199603000-00008

[3] Wang, S., Zhang, Z. and Xia, L. (2010) Systematic Evaluation of a Tissue-Engineered Bone for Maxillary Sinus Augmentation in Large Animal Canine Model. Bone, 46, 91-100. http://dx.doi.org/10.1016/j.bone.2009.09.008

[4] Zhang, W., Wang, X., Wang, S., et al. (2011) The Use of Injectable Sonication-Induced Silk Hydrogel for VEGF(165) and BMP-2 Delivery for Elevation of the Maxillary Sinus Floor. Biomaterials, 32, 9415-9424. http://dx.doi.org/10.1016/j.biomaterials.2011.08.047

[5] Block, M.S., Ducote, C.W. and Mercante, D.E. (2012) Horizontal Augmentation of Thin Maxillary Ridge with Bovine Particulate Xenograft Is Stable during 500 Days of Follow-Up: Preliminary Results of 12 Consecutive Patients. Journal of Oral and Maxillofacial Surgery, 70, 1321-1330. http://dx.doi.org/10.1016/j.joms.2012.01.026

[6] Barone, A., Toti, P., Piattelli, A., et al. (2014) Extraction Socket Healing in Humans after Ridge Preservation Techniques: A Comparison between Flapless and Flapped Procedure in a Randomized Clinical Trial. Journal of Periodontology, 85, 14-23.

[7] Sbordone, C., Toti, P., Guidetti, F., et al. (2012) Volume Changes of Iliac Crest Autogenous Bone Grafts after Vertical and Horizontal Alveolar Ridge Augmentation of Atrophic Maxillas and Mandibles: A 6-Year Computerized Tomographic Follow-Up. Journal of Oral and Maxillofacial Surgery, 70, 2559-2565. http://dx.doi.org/10.1016/j.joms.2012.07.040

[8] Cordaro, L., Torsello, F., Miuccio, M.T., et al. (2011) Mandibular Bone Harvesting for Alveolar Reconstruction and Implant Placement: Subjective and Objective Cross-Sectional Evaluation of Donor and Recipient Site up to 4 Years. Clinical Oral Implants Research, 22, 1320-1326. http://dx.doi.org/10.1111/j.1600-0501.2010.02115.x

[9] Nissan, J., Gross, O., Mardinger, O., et al. (2011) Post-Traumatic Implant-Supported Restoration of the Anterior Maxillary Teeth Using Cancellous Bone Block Allografts. Journal of Oral and Maxillofacial Surgery, 69, e513-e518. http://dx.doi.org/10.1016/j.joms.2011.08.002

[10] Wakimoto, M., Matsumura, T., Ueno, T., et al. (2012) Bone Quality and Quantity of the Anterior Maxillary Trabecular Bone in Dental Implant Sites. Clinical Oral Implants Research, 23, 1314-1319. http://dx.doi.org/10.1111/j.1600-0501.2011.02347.x

[11] Nkenke, E., Schultze-Mosgau, S., Radespiel-Troger, M., et al. (2001) Morbidity of Harvesting of Chin Grafts: A Prospective Study. Clinical Oral Implants Research, 12, 495-502. http://dx.doi.org/10.1034/j.1600-0501.2001.120510.x

[12] Dasmah, A., Thor, A., Ekestubbe, A., et al. (2012) Particulate vs. Block Bone Grafts: Three-Dimensional Changes in Graft Volume after Reconstruction of the Atrophic Maxilla, a 2-Year Radiographic Follow-Up. Journal of Cranio-Maxillofacial Surgery, 40, 654-659.

[13] Nyström, E., Legrell, P.E., Forssell, A., et al. (1995) Combined Use of Bone Grafts and Implants in the Severely Resorbed Maxilla. Postoperative Evaluation by Computed Tomography. International Journal of Oral and Maxillofacial Surgery, 24, 20-25. http://dx.doi.org/10.1016/S0901-5027(05)80851-3

[14] Her, S., Kang, T. and Fien, M.J. (2012) Titanium Mesh as an Alternative to a Membrane for Ridge Augmentation. Journal of Cranio-Maxillofacial Surgery, 70, 803-810. http://dx.doi.org/10.1016/j.joms.2011.11.017 
[15] Thor, A. (2002) Reconstruction of the Anterior Maxilla with Platelet Gel, Autogenous Bone, and Titanium Mesh: A Case Report. Clinical Oral Implants Research, 4, 150-155. http://dx.doi.org/10.1111/j.1708-8208.2002.tb00166.x

[16] Esposito, M., Hirsch, J.M., Lekholm, U. and Thomsen, P. (1998) Biological Factors Contributing to Failures of Osseointegrated Oral Implants, (I). Success Criteria and Epidemiology. European Journal of Oral Sciences, 106, 527-551. http://dx.doi.org/10.1046/j.0909-8836..t01-2-.x

[17] Cosyn, J., Sabzevar, M.M. and De Bruyn, H. (2012) Predictors of Inter-Proximal and Midfacial Recession Following Single Implant Treatment in the Anterior Maxilla: A Multivariate Analysis. Journal of Clinical Periodontology, 39, 895-903. http://dx.doi.org/10.1111/j.1600-051X.2012.01921.x

[18] Ishikawa, T., Salama, M., Funato, A., Kitajima, H., Moroi, H., Salama, H. and Garber, D. (2010) Three-Dimensional Bone and Soft Tissue Requirements for Optimizing Esthetic Results in Compromised Cases with Multiple Implants. International Journal of Periodontics \& Restorative Dentistry, 30, 503-511.

[19] Dasmah, A., Thor, A., Ekestubbe, A., Sennerby, L. and Rasmusson, L. (2013) Marginal Bone-Level Alterations at Implants Installed in Block versus Particulate Onlay Bone Grafts Mixed with Platelet-Rich Plasma in Atrophic Maxilla. A Prospective 5-Year Follow-Up Study of 15 Patients. Clinical Implant Dentistry and Related Research, 15, 7-14. http://dx.doi.org/10.1111/j.1708-8208.2011.00377.x

[20] Johannsen, A., Westergren, A. and Johannsen, G. (2012) Dental Implants from the Patients Perspective: Transition from Tooth Loss, through Amputation to Implants-Negative and Positive Trajectories. Journal of Clinical Periodontology, 39, 681-687. http://dx.doi.org/10.1111/j.1600-051X.2012.01893.x

[21] Ortega-Martinez, J., Perez-Pascual, T., Mareque-Bueno, S., Hernández-Alfaro, F. and Ferrés-Padró, E. (2012) Immediate Implants Following Tooth Extraction. A Systematic Review. Medicina Oral, Patología Oral y Cirugía Bucal, 17, e251-e261. http://dx.doi.org/10.4317/medoral.17469

[22] Lundgren, S., Sjostrom, M., Nystrom, E. and Sennerby, L. (2008) Strategies in Reconstruction of the Atrophic Maxilla with Autogenous Bone Grafts and Endosseous Implants. Periodontology 2000, 47, 143-161. http://dx.doi.org/10.1111/j.1600-0757.2008.00265.x

[23] Nyström, E., Lundgren, S., Gunne, J. and Nilson, H. (1997) Interpositional Bone Grafting and Le Fort I Osteotomy for Reconstruction of the Atrophic Edentulous Maxilla. A Two-Stage Technique. International Journal of Oral and Maxillofacial Surgery, 26, 423-427. http://dx.doi.org/10.1016/S0901-5027(97)80006-9

[24] Cosyn, J. and De Rouck, T. (2009) Aesthetic Outcome of Single-Tooth Implant Restorations Following Early Implant Placement and Guided Bone Regeneration: Crown and Soft Tissue Dimensions Compared with Contralateral Teeth. Clinical Oral Implants Research, 20, 1063-1069. http://dx.doi.org/10.1111/j.1600-0501.2009.01746.x

[25] Davis, W.H., Martinoff, J.T. and Kaminishi, R.M. (1984) Long-Term Follow up of Transoral Rib Grafts for Mandibular Atrophy. Journal of Oral and Maxillofacial Surgery, 42, 606-609. http://dx.doi.org/10.1016/0278-2391(84)90092-2

[26] Donovan, M.G., Dickerson, N.C., Hellstein, J.W. and Hanson, L.J. (1993) Autologous Calvarial and Iliac Onlay Bone Grafts in Miniature Swine. Journal of Oral and Maxillofacial Surgery, 51, 898-903. http://dx.doi.org/10.1016/S0278-2391(10)80112-0

[27] von Arx, T. and Buser, D. (2006) Horizontal Ridge Augmentation Using Autogenous Block Grafts and the Guided Bone Regeneration Technique with Collagen Membranes: A Clinical Study with 42 Patients. Clinical Oral Implants Research, 17, 359-366. http://dx.doi.org/10.1111/j.1600-0501.2005.01234.X

[28] Kühl, S., Götz, H., Hansen, T., Kreisler, M., Behneke, A., Heil, U., Duschner, H. and d’Hoedt, B. (2010) Three-Dimensional Analysis of Bone Formation after Maxillary Sinus Augmentation by Means of Microcomputed Tomography: A Pilot Study. International Journal of Oral \& Maxillofacial Implants, 25, 930-938.

[29] Bhandari, S. and Chaturvedi, R. (2012) Immediate Natural Tooth Pontic: A Viable yet Temporary Prosthetic Solution: A Patient Reported Outcome. Indian Journal of Dental Research, 23, 59-63. http://dx.doi.org/10.4103/0970-9290.99040

[30] Lin, K.Y., Bartlett, S.P., Yaremchuk, M.J., Fallon, M., Grossman, R.F. and Whitaker, L.A. (1990) The Effect of Rigid Fixation on the Survival of Onlay Bone Grafts: An Experimental Study. Plastic \& Reconstructive Surgery, 86, 449-456. http://dx.doi.org/10.1097/00006534-199009000-00010

[31] Hellem, S., Astrand, P., Stenstrom, B., Engquist, B., Bengtsson, M. and Dahlgren, S. (2003) Implant Treatment in Combination with Lateral Augmentation of the Alveolar Process: A 3-Year Prospective Study. Clinical Implant Dentistry and Related Research, 5, 233-240. http://dx.doi.org/10.1111/j.1708-8208.2003.tb00206.x 


\section{Abbreviations}

IPS, implant placement sites; NIPS, non-implant placement sites; ACBW, alveolar crestal bone width; ABBW, alveolar midway bone width; $\mathrm{ABH}$, alveolar bone height; T0, 1 month before surgery; T1, immediately after surgery; T2, 3 to 4 months after surgery; T3, 6 to 7 months after surgery before implant placement; T4, 12 to 13 months after surgery; T5, the longest postoperative follow-up point; T1-T0, the bone augmentation volume from $\mathrm{T} 0$ to T1; T1-T2, the bone resorption volume from T1 to T2; T2-T3, the bone resorption volume from T2 to T3; T3-T4, the bone resorption volume from T3 to T4, T4-T5: the bone resorption volume from T4 to T5; ${ }^{*} \mathrm{P}<0.05$. 